Pure and Applied Mathematics Quarterly

Volume 7, Number 1

(Special Issue: In honor of

Frederick W. Gehring, Part 1 of 2)

$121-138,2011$

\title{
A Set Containing Rectifiable Arcs QC-locally But Not QC-globally
}

\author{
Christopher J. Bishop \\ To Professor F.W. Gehring on his 80th Birthday.
}

\begin{abstract}
We construct a Sierpinski carpet $E \subset \mathbb{R}^{2}$ of area zero and a $K_{0}>1$ with the property that every $K_{0}$-quasiconformal image of $E$ contains rectifiable curves, but such that $E$ has some quasiconformal image containing no non-constant rectifiable curves.
\end{abstract}

Keywords: $A_{1}$ weight, quasiconformal mappings, Jacobian problem, rectifiable curves, Sierpinski carpet.

\section{INTRODUCTION}

In [1] I constructed an $A_{1}$ weight on the plane which is not comparable to the Jacobian of any quasiconformal mapping, answering a question that had been posed in various places (e.g., [4], [7], [8], [9] and [13]). The main idea was to construct a compact set $E$ and a weight $w$ that blows up on $E$, but such that the image of $E$ under any quasiconformal map $f$ with Jacobian comparable to $w$ would have to contain rectifiable curve $\gamma$. Then the Jacobian of $f^{-1}$ would vanish on $\gamma$, which implies $f^{-1}(\gamma)$ is a point, a contradiction.

This result leads to various natural questions which are posed at the end of [1], e.g., is there a set $E$ of zero area such that any quasiconformal image of $E$ contains

Received August 17, 2006.

2000 Mathematics Subject Classification. Primary: 30C62 Secondary: 31A05, 53A10.

The author is partially supported by NSF Grant DMS 01-03626. 
a rectifiable curve? Is it possible that this can happen for small quasiconformal constants, but not for large ones? In this note, we answer the last question with:

Theorem 1.1. There is a compact set $E \subset \mathbb{R}^{2}$ of area zero and a $K_{0}>1$ so that every $K_{0}$-quasiconformal image of $E$ contains rectifiable curves, but there is some quasiconformal image of $E$ which contains no rectifiable curves.

Corollary 1.2. There is a compact set $E$ of zero area and a $K_{0}>1$ so that no function $w$ such that $w(z) \rightarrow \infty$ as $z \rightarrow E$ can be comparable to the Jacobian of a $K_{0}$-quasiconformal map.

Suppose $E \subset \mathbb{R}^{2}$ and let $\mathrm{QC}_{\mathrm{K}}(E)$ denote the collection of $K$-quasiconformal images of $E$. We say that $E$ has a property QC-globally it holds for all sets in $\cup_{K \geq 1} \mathrm{QC}_{\mathrm{K}}(E)$ and say it holds QC-locally if it holds for all sets in $\mathrm{QC}_{\mathrm{K}}(E)$ when $K$ is close enough to 1 . Thus Theorem 1.1 can be restated by saying there is a compact set of zero area which contains rectifiable curves QC-locally but not QC-globally.

The set we construct will be a type of Sierpinski carpet where $E=\cap_{n} E_{n}$ with $E_{1} \supset E_{2} \supset \ldots$, and where $E_{n+1}$ is obtained by writing $E_{n}$ as a union of squares and then replacing each square with one of two types of "building blocks". Each type is a polygonal region with a finite number of square holes removed. Both types have the property that each hole is surrounded by a thick annulus. This property implies that the set, and any small quasiconformal deformation of it, contains rectifiable curves. The first type of building block is fairly simple, used frequently, and has enough holes to make sure the the area of $E_{n}$ tends to zero. The second type of block is more complicated and occurs more sparsely. It has its holes arranged so that any sufficiently long line segment that hits the block must come close to a hole with precise estimates; using this property and a characterization of tangents in terms of Peter Jones' $\beta$ 's we will construct a quasiconformal image of $E$ which contains no rectifiable subset.

This paper is a sequel to [1] which was motivated by lectures and conversations at the May 2005 Ahlfors-Bers colloquium in Ann Arbor. Many thanks to the organizers for an exciting and informative conference and to Juha Heinonen and Mario Bonk for helpful remarks on the problems considered here. Also thanks to Yuval Peres for the statement and proof of Lemma 2.3 and Joe Mitchell for related comments. I thank the referee for a careful reading of the manuscript and 
especially for pointing out an error in the original proof of Theorem 1.1 (fixed in the current version by an application of the strong law of large numbers).

In Section 2 we will review some results needed in the construction. In Section 3 we will prove Theorem 1.1.

\section{Preliminaries}

In this section we discuss some results that will be needed in the construction of the set $E$. These deal with conditions for showing a set either does or does not contain a rectifiable curve.

2.1. A sufficient condition for having rectifiable subcurves. The following criteria for containing a rectifiable curve is taken from [1].

Lemma 2.1. Suppose that $E_{0} \supset E_{1} \supset E_{2} \supset E_{3} \ldots$ are compact sets and $E=$ $\cap E_{n}$. Suppose there is a $K<\infty, C>0$ and sequences of positive numbers $\left\{P_{n}\right\}$, $\left\{Q_{n}\right\}$ so that the following holds. For $n=1,2, \ldots$, suppose $E_{n}=\cup_{k} \Omega_{k, n}=E_{n-1} \backslash$ $\cup_{k, j} W_{j, k, n}$ (disjoint except for boundaries) where $\Omega_{k, n}$ consists of a closed $K$ quasidisk $W_{k, n}$ with a finite number of open $K$-quasidisks $W_{j, k, n}$ removed (called the omitted regions). Moreover, for each $j, k$ assume there is a collection of disjoint disks $\left\{D_{n}\right\}=\left\{D\left(x_{n}, r_{n}\right)\right\}$ so that with the properties that

(1) $\operatorname{dist}\left(D_{n}, \partial W_{k, n}\right) \geq C \operatorname{diam}\left(W_{k, n}\right)$.

(2) $W_{j, k, n} \subset D\left(x_{n}, r_{n} / Q_{n}\right) \subset D\left(x_{n}, P_{n} r_{n}\right) \subset W_{j, k}$.

If $\sum_{n} P_{n}^{-2}<\infty$ and $\sum_{n} Q_{n}^{-3}<\infty$, then $E$ contains rectifiable curves.

The main idea of the proof is to start with a line segment in $E_{0}$ with endpoints on the boundary. In general if we are given a polygonal curve in $E_{n}$ with its vertices all on the boundaries of $n$th generation squares then we perturb the curve to remain in $E_{n+1}$ with only a small gain in length. Taking the limit as $n \rightarrow \infty$ gives a rectifiable curve in $E$. The details are discussed in [1].

2.2. A sufficient condition for having no rectifiable subsets. To show a set $E$ contains no rectifiable curves, we will use the fact that every rectifiable curve has tangents almost everywhere and that this holds in a quantifiable way. 
Suppose $\Gamma$ is a compact set in $\mathbb{R}^{2}$. For $x \in \Gamma$ and $t>0$ define $\beta(x, t)$ as

$$
\beta(x, t)=\inf _{L}\left\{\sup \frac{\operatorname{dist}(z, L)}{t}: z \in \Gamma \cap D(x, t)\right\}
$$

where the infimum is taken over all lines $L$ passing through $D(x, t)$. These are called Peter Jones' $\beta$ 's; he used them to characterize connected sets of finite length in the plane (see [2], [3],[10], [11], [12]). If a curve $\Gamma$ has a tangent at a point $x$ then clearly $\beta(x, t) \rightarrow 0$ as $t \rightarrow 0$. We shall use the following, more quantitative, version of this fact (Theorem 3 of [3] or Theorem X.2.5 in [6]).

Theorem 2.2. Suppose $\Gamma$ is a compact, connected set. Then except for a set of zero 1-dimensional measure, $x \in \Gamma$ is a tangent point of $\Gamma$ iff

$$
\int_{0}^{1} \beta^{2}(x, t) \frac{d t}{t}<\infty
$$

We will use this in a slightly different form.

Lemma 2.3. Suppose $\Gamma$ is a rectifiable curve and $x \in \Gamma$. Suppose that associated to each $x$ there are sequences $\left\{a_{n}(x)\right\} \subset[0,1]$ and $\left\{t_{n}(x)\right\} \searrow 0$ with $t_{n+1}(x)<\frac{1}{2} t_{n}(x)$ so that any line segment of length $t_{n}(x)$ with $x$ as one endpoint also contains a point $y$ with $\operatorname{dist}(y, E) \geq a_{n}(x) t_{n}(x)$. Then for almost every $x \in \Gamma, \sum_{n} a_{n}^{2}(x)<\infty$.

Proof. Suppose $x \in \Gamma$ and $\left\{t_{n}\right\}$ is as in the lemma. Without loss of generality we assume the scales are all less than $\operatorname{diam}(\Gamma)$. Then for $t_{n} / 2<r \leq t_{n}, \beta(x, r) \geq$ $a_{n} / 4$, for otherwise $\Gamma$ would be trapped inside a $a_{n} t / 2$-wide strip inside $D\left(x, t_{n}\right)$ which is impossible since $\Gamma$ has larger diameter. Hence $\beta(x, r) \geq a_{n} / 2$ for $t_{n} / 2<$ $r<t_{n}$ and so

$$
\int_{t_{n} / 2}^{t_{n}} \beta^{2}(x, r) d r / r \geq \frac{\ln 2}{16} a_{n}^{2}
$$

Thus Lemma 2.2 implies $\sum a_{n}^{2}$ converges for a.e. $x \in \Gamma$.

We shall use this later as follows. We will construct a set $E$ and a quasiconformal map $f$ and will want to prove that $f(E)$ contains no rectifiable curves. We will assume that it does contains such a curve $\Gamma$ and prove that there is a subset $X \subset \Gamma$ of positive length so that for every $x \in X$ we can choose $\left\{a_{n}\right\}$ and $\left\{t_{n}\right\}$ as in Lemma 2.3 so that $\sum a_{n}^{2}=\infty$ almost everywhere on $X$. This contradicts Lemma 2.3 and hence shows $f(E)$ contains no rectifiable arcs. 
2.3. How far can you see in a forest? Suppose we stand in forest with tree trunks of radius $\epsilon$ and no two trees centered closer than unit distance apart. How far can we see? If the trees are placed on the usual square lattice we can see forever by looking horizontally at height $1 / 2$. It is also easy to show that you can always see at least distance $\sim 1 / \epsilon$ from some point. Can we arrange the trees so that we can never see farther than some distance $L<\infty$, no matter where we stand or what direction we look in? How is $L$ bounded in terms of $\epsilon$ ?

We will say the set $E \subset \mathbb{R}^{2}$ is an $(\epsilon, C, \alpha)$-forest for a set $X \subset \mathbb{R}^{2}$ if there is an $r>0$ so that

(1) Any two points of $E$ are separated by at least $r$,

(2) any line segment of length $L \geq C \epsilon^{-\alpha} r$ which hits $X$ comes within $\epsilon r$ of some point of $E$.

The following result and proof was provided to me by Yuval Peres. It shows there is a forest in the plane where visibility from any point is less than $O\left(\epsilon^{-4}\right)$. A similar example was suggested independently by Joe Mitchell.

Lemma 2.4 (Yuval Peres, personal communication). There is a $C<\infty$ so that for any $\epsilon \in(0,1]$ there is a set $E \subset \mathbb{R}^{2}$ which is a $(\epsilon, C, 4)$-forest.

Proof. We will first build a set which hits lines which are close to horizontal, i.e. line segments of the form

$$
I=\left\{(x, y): y=b x+d \text { with } x_{0} \leq x \leq x_{0}+L / 2\right\},
$$

for some real $d$ and $b$ with $|b|<1$. We may assume $x_{0}$ is an integer.

The set $E$ will consist of the union of a horizontal translate of the usual square lattice $\mathbb{Z}^{2}$ and a copy of this lattice under a vertical irrational shear. More precisely, let $E=S_{0} \cup S_{1}$, where

$$
S_{0}=\mathbb{Z}^{2}+\left(\frac{1}{2}, 0\right), \quad S_{1}=\{(m, n+m g): m, n \in \mathbb{Z}\} .
$$

Here $g=(1+\sqrt{5}) / 2 \approx 1.618 \ldots$ is the golden mean. See Figure 1 . Obviously, any two points of $E$ are at least distance $1 / 2$ apart, so we can take $r=1 / 2$.

We use the golden mean $g$ because it satisfies the estimate

$$
\|g q\| \geq c / q
$$




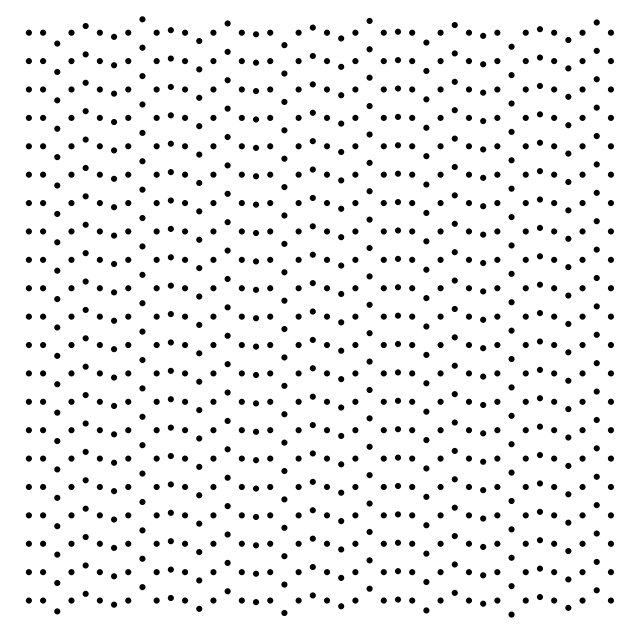

Figure 1. The Peres forest for $\epsilon=.1$ in the square $[0,20]^{2}$.

for every integer $q$, where for $x \in \mathbb{R}^{d}$ we write $\|x\|=\operatorname{dist}\left(x, \mathbb{Z}^{d}\right)$. Liouville's argument for this (taken from Chapter VI of Cassels' book [5]) is as follows. The number $g$ is a root of $f(x)=x^{2}-x-1$. The derivative $f^{\prime}(x)=2 x-1$ is bounded by $2\left(g+\frac{1}{2}\right)-1=1+\sqrt{5}=c$ on the interval $\left[g-\frac{1}{2}, g+\frac{1}{2}\right]$ and hence $\left|f\left(\frac{p}{q}\right)\right| \leq \sqrt{5}\left|g-\frac{p}{q}\right|$. For any integer $q, q^{2} f\left(\frac{p}{q}\right)$ is a non-zero integer and hence $\left|q^{2} f\left(\frac{p}{q}\right)\right| \geq 1$ and hence $\left|f\left(\frac{p}{q}\right)\right| \geq q^{-2}$. Moreover, for any integer $q$ the $p$ which minimizes $\left|g-\frac{p}{q}\right|$ satisfies $\left|g-\frac{p}{q}\right| \leq 12$. For such a choice

$$
\|g q\|=|g q-p|=q\left|g-\frac{p}{q}\right| \geq \frac{q}{1+\sqrt{5}} f\left(\frac{p}{q}\right) \geq \frac{q}{(1+\sqrt{5}) q^{2}},
$$

as desired.

Next, we have to show that there is a $C<\infty$ so that any line segment $I$ of the form (2.1) of length $L \geq C \epsilon^{-4}$ comes within $\epsilon$ of some element of $E$. By the pigeonhole principle there is an integer $q \in\left(0,2 \epsilon^{-2}\right)$ so that $\|(q g, q b)\|<\epsilon$. Moreover, $\|q g\|>c / q \geq 2 c \epsilon^{2}$, so by the triangle inequality we have either

$$
\|q b\|>c \epsilon^{2}
$$

or

$$
\|q(g-b)\|>c \epsilon^{2}
$$

First suppose (2.2) holds. Then the numbers $\left\{j q b: 0<j \leq \frac{2}{c} \epsilon^{-2}\right\}$ are $\epsilon$-dense mod one (since the step between $j$ and $j+1$ is at most $\epsilon$ and at least $c \epsilon^{2}$ ), so we 
can choose positive $j \leq 2 /\left(c \epsilon^{2}\right)$ such that $m=x_{0}+j q$ satisfies $\left\|b m+d+\frac{1}{2} b\right\|<\epsilon$. Hence $\left|\left(m+\frac{1}{2}, n\right)-\left(m+\frac{1}{2}, b m+\frac{1}{2} b+d\right)\right|<\epsilon$ and note that $x_{0}<m \leq x_{0}+L / 2$. Thus there is a point of $I$ close to $S_{0}$.

Otherwise (2.3) holds, so the multiples $\left\{j q(g-b): 0<j \leq \frac{2}{c} \epsilon^{-2}\right\}$ are $\epsilon$-dense. Thus we can choose $m=x_{0}+j q$ so that $\|m(b-g)+d\|<\epsilon$. Hence the distance from $(m, m b+d) \in I$ to $S_{1}$ is at most $\epsilon$.

Now for the general case. Suppose we could divide the plane into two disjoint sets $\mathbb{R}^{2}=A \cup B$ with the property that there is a $M<\infty$ so that any line segment $I$ of length $\geq M L$ satisfies

$$
|I \cap A(2)| \geq L,|I \cap B(2)| \geq L,
$$

where $A(r)=\{z \in A: \operatorname{dist}(z, \partial A) \geq r\}$, and similarly for $B(r)$. Then taking $(E \cap A(1)) \cup(i E \cap B(1))$ would be a $(\epsilon, M C, 4)$-forest. Such a decomposition of the plane is not hard to produce, e.g., see the "stairsteps" illustrated in Figure 2 .

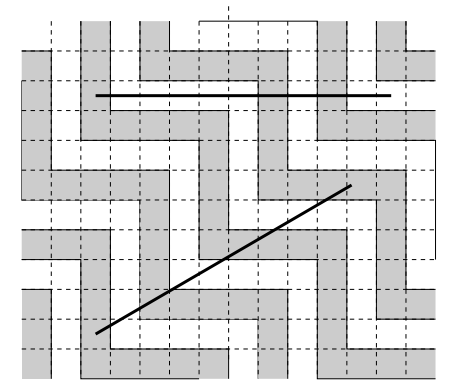

Figure 2. A division of the plane into two sets (shaded and white) so that any sufficiently long segments hits both sets away from their boundaries.

We will want to use forests which are restricted to certain domains. Suppose $\Omega$ is a bounded quasidisk with $d=\operatorname{diam}(\Omega)$. We will need a finite set $E$ in $\Omega$ so that any sufficiently long line segment that hits $\Omega$ (away from its boundary) will come close to a point of $E$. To be more precise, given some $a>0$, we will say a finite set $E$ is a $(a, \epsilon)$-forest for $\Omega$ if

(1) $E \subset \Omega(a d / 2)$, 
(2) points of $E$ are separated by strictly more than $C a d / 2 \epsilon^{4}$ ( $C$ is the constant from Lemma 2.4),

(3) Any line segment $L$ of length $\geq a d$ which hits $\Omega(a d)$ comes strictly within distance $\epsilon^{5}$ of some point of $E$.

It is immediate from Lemma 2.4 that such a forest always exists (just intersect the forest constructed there with $\Omega(a d / 2))$.

Also note that if $E$ is an $(a, \epsilon)$-forest for $\Omega$ and $f$ is conformal, linear map, then $f(E)$ is an $(a, \epsilon)$-forest for $\Omega^{\prime}=f(\Omega)$. Because of the strict inequalities in the definition of a forest for $\Omega$, we can slightly perturb the points of $E$ and still have a forest with the same constants. We will use this observation several times in Section 3.5.

2.4. The strong law of large numbers. The strong law of large numbers can be stated as follows: if $\left\{f_{n}\right\}$ is a bounded, orthogonal sequence of functions in $L^{2}([0,1], d x)$ then $\lim _{n \rightarrow \infty} \frac{1}{n} \sum_{k=1}^{n} f_{k}(x)=0$ for a.e. $x$. We shall make use of this in the following form (which is stronger version of the Borel-Cantelli lemma). Let $\chi_{E}$ denote the characteristic function of $E$, i.e., $\chi_{E}$ is 1 on $E$ and 0 off $E$.

Lemma 2.5. Suppose $\left\{E_{n}\right\} \subset[0,1]$ is a sequence of compact sets, each of which is a finite union of intervals. Suppose also that if $I$ is a component of $E_{n}$ or $[0,1] \backslash E_{n}$, and $m>n$ then $\left|E_{m} \cap I\right| \geq c_{1}|I|$ for some $c_{1}>0$, independent of $n$. Then for almost every $x \in[0,1],\left\{k: x \in E_{k}\right\}$ has positive lower density in the integers, i.e., $\sum_{k=1}^{n} \chi_{E_{k}}(x) \geq \frac{1}{2} c_{1} n$, for $n$ sufficiently large.

Proof. We define a bounded sequence of orthogonal functions $\left\{f_{n}\right\}$ on $[0,1]$ which are piecewise constant on the components of $E_{n}$ and $E_{n}^{c}$. First let $f_{1}=\chi_{E_{1}}-\left|E_{1}\right|$. Then $f_{0}$ has mean value zero over $[0,1]$. In general, suppose $I$ is a component interval of either $E_{n-1}$ or its complement in $[0,1]$. Define $f_{n}(x)$ on $I$ as $\chi_{E_{n}}(x)-$ $\left|E_{n} \cap I\right| /|I|$. Then $f_{n}$ has mean value zero over $I$ and since $f_{n-1}$ is constant on $I$, we deduce that $f_{n}$ and $f_{n-1}$ are orthogonal. Similarly $f_{n}$ is orthogonal to every $f_{k}$ for $k<n$.

Since $\left|E_{n} \cap I\right| \leq|I|$, we are subtracting a constant between 0 and 1 from $\chi_{E_{n}}$, so the function $f_{n}$ is clearly bounded between -1 and 1 . Thus the strong law of 
large numbers applies and we deduce that

$$
\lim _{n \rightarrow \infty} \frac{1}{n} \sum_{k=1}^{n} f_{n}(x)=0,
$$

for a.e. $x$. Unwinding the definitions, this is the same as

$$
\lim _{n \rightarrow \infty} \frac{1}{n}\left(\sum_{k=1}^{n} \chi_{E_{n}}(x)-\frac{\left|E_{n} \cap I\right|}{|I|}\right)=0,
$$

which implies

$$
\sum_{k=1}^{n} \chi_{E_{n}}(x) \geq n c_{1}+o(n) \geq \frac{1}{2} c_{1} n,
$$

if $n$ is large enough. This is the claim about positive density.

Corollary 2.6. With notation as above, suppose that for each $x,\left\{a_{n}(x)\right\}$ is a decreasing sequence of positive reals such that $\sum_{n} a_{n}(x)=\infty$. Then for a.e. $x \in[0,1], \sum_{n} a_{n}(x) \chi_{E_{n}}(x)=\infty$.

Proof. Since $\left\{a_{n}\right\}$ is decreasing, the sum of any $N$ terms from an interval of integers is bounded below by the sum of the last $N$ terms. Choose $A$ large and $\epsilon>0$ small so that $\frac{1}{2} c_{1} A-1>\epsilon A$ for large enough $n$. Then

$$
\begin{aligned}
\sum_{n=1}^{\infty} a_{n}(x) \chi_{E_{n}}(x) & =\sum_{k=1}^{\infty} \sum_{A^{k-1} \leq n<A^{k}-1} a_{n}(x) \chi_{E_{n}}(x) \\
& \geq \sum_{k=1}^{\infty} \sum_{(1-\epsilon) A^{k} \leq n<A^{k}-1} a_{n}(x) \\
& \geq \sum_{k=1}^{\infty} \frac{\epsilon}{A} \sum_{(1-\epsilon) A^{k} \leq n<(1-\epsilon) A^{k+1}-1} a_{n}(x) \\
& \geq \frac{C \epsilon}{A} \sum_{n>A} a_{n}(x) \\
& =\infty,
\end{aligned}
$$

as desired.

2.5. Approximation by Lipschitz graphs. An $\epsilon$-Lipschitz function on the real line is one that satisfies $|f(x)-f(y)| \leq \epsilon|x-y|$. An $\epsilon$-Lipschitz graph in the plane is any image under a Euclidean similarity of a curve of the form $\Gamma=\{(x, f(x)): x \in I\}$ where $I$ is an interval and $f$ is $\epsilon$-Lipschitz. When $\epsilon$ is 
close to zero, then $\Gamma$ is close to a straight line segment. Lipschitz graphs are rectifiable, and although not every rectifiable curve is a Lipschitz graph, every rectifiable curve can be approximated by a Lipschitz graph in the following sense.

Lemma 2.7. Suppose $\Gamma$ is a rectifiable curve in $\mathbb{R}^{2}$. Then for any $\epsilon>0$ there is an $\epsilon$-Lipschitz graph $\gamma$ so that $\gamma \cap \Gamma$ has positive length.

This is a standard result, but we repeat the argument briefly. Almost every point of a rectifiable curve $\Gamma$ is the tip of cone with angle $\pi-\epsilon$ which is disjoint from the curve. We may assume the angle of the axis of the cone is rational, as is its angle and diameter. Thus there is a set of positive measure on $\Gamma$ for which the same choice of angle, axis direction and diameter all work. The union of the corresponding cones defines an $\epsilon$-Lipschitz graph which hits $\Gamma$ in positive measure.

\section{The CONSTRUCTION}

The set $E$ satisfying Theorem 1.1 will be constructed by an iterative process involving two types of replacements. We start with a description of each type.

3.1. Type I pieces. Suppose $L$ and $M$ are positive integers and $M$ is odd. A type I $(L, M)$-piece is built as follows. Given a square $Q$ divide it into 36 equal sized subsquares called type 1 . The subcollection of type 1 subsquares that are adjacent to $\partial Q$ are called the boundary squares. Divide each of the 16 non-boundary type 1 squares into $L^{2}$ equal subsquares (type 2) and divide each of these into $M^{2}$ equal subsquares (type 3 ). For each type 2 square remove the unique type 3 square containing its center. This gives a region $\Omega(Q, L, M)$ which is the type I piece associated to $Q$. See Figure 3. Note that each of the removed squares has diameter $\simeq \operatorname{diam}(Q) / L M$ and the total area removed is $\simeq \operatorname{area}(Q) / M^{2}$.

3.2. Type II pieces. A type II piece is a little more complicated and will depend on a single positive integer parameter $N$. Given a square $Q$ we replace it with the region bounded by the 20-gon shown in Figure 4 . We will call this a "bent square" or a "tile". The shape is a square with rectangles added along the top and right edges and removed along the bottom and left edges. More precisely, take the square $Q$ and break it into $20^{2}=400$ equal subsquares. Add a $5 \times 5$ 
A Set Containing Rectifiable Arcs QC-locally But Not QC-globally 131

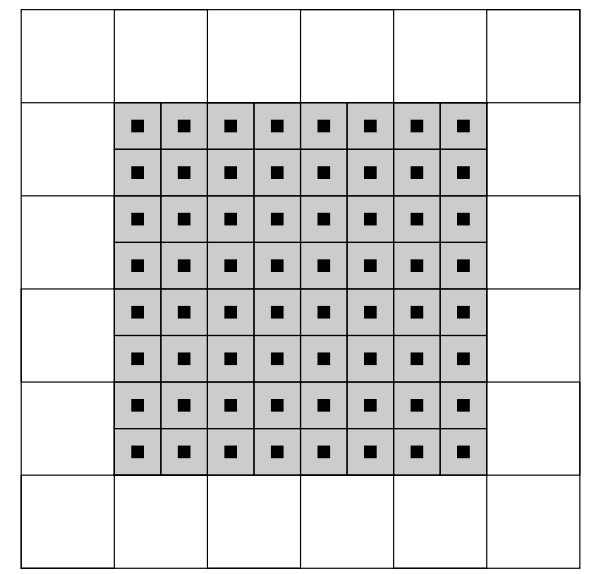

Figure 3. A type I piece for $L=2, M=3$. The boundary squares are white, the non-boundary squares are gray and the omitted squares are black.

blocks to the top and right edges and remove them along the left and bottom edges as shown in Figure 4. Divide the resulting region $R$ into 400 equal sized squares. $S_{1}$ is the union of subsquares which do not touch $\partial R$ and are shaded light gray n Figure 5. $S_{2}$ is the union of subsquares of $S_{1}$ which do not touch $\partial S_{1}$. These are shaded darker gray.

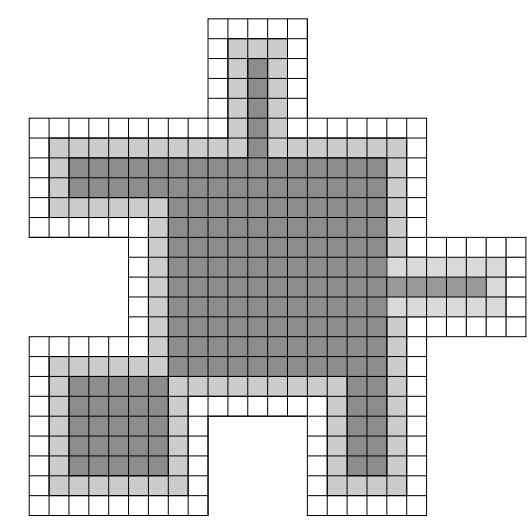

Figure 4. The definition of $R$ (whole figure), $S_{1}$ (all gray squares), $S_{2}$ (dark gray only) and the boundary squares (white only). 
Note that if $Q$ and $Q^{\prime}$ are disjoint squares sharing an edge, then the corresponding bent squares are also disjoint and share a boundary arc. See Figure 5.

Lemma 3.1. There is a $C>0$ so that the following holds. Consider the collection of bent tiles $\left\{Q_{j}\right\}$ corresponding to the usual integer grid. Let $W$ be the corresponding union of interior squares (everything of the form $S_{1}$ in every tile, i.e., the gray region in Figures 4 and 5). Then any line segment $L$ of length 2 contains a subsegment $J$ of length $\geq C$ inside $W$.

The proof is evident from Figure 5, since the "channels" formed by the white and light gray regions do not contain any line segments of length 2. Any such segment must therefore hit some point colored dark gray. Such a point is contained in a subsegment of length at least $1 / 20$, i.e., the side length of a light gray square.

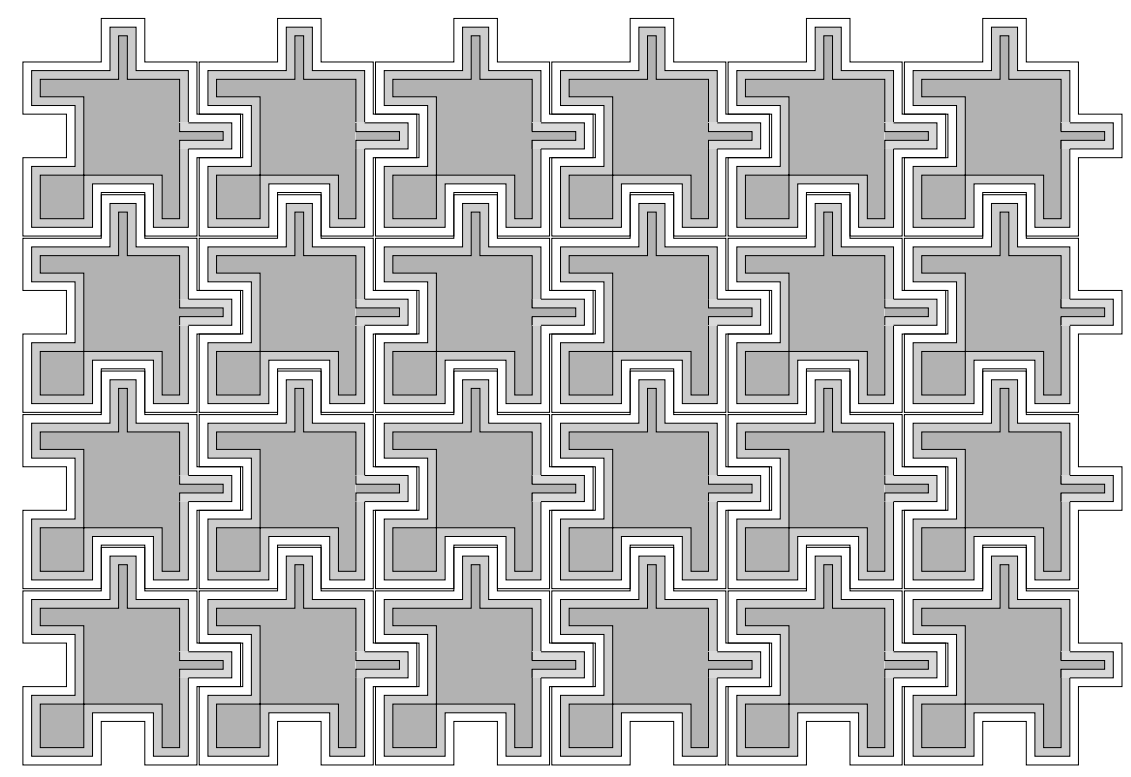

Figure 5. The replacement pieces fit together to tile a region. When we remove the boundary squares of each piece we are left with the shaded region. Any long enough length line segment must hit the dark gray region and hence spends a certain fraction of its length in the gray region. 
We actually need to use a slight generalization of Lemma 3.1. Use the same notation as before.

Lemma 3.2. There is an $\epsilon>0$ and $C<\infty$ so that the following holds. If $Q$ is an individual bent tile, $f$ is a quasiconformal map of the plane so that $|f(z)-z| \leq \epsilon$ for all $z$ and $\gamma$ is an $\epsilon$-Lipschitz graph of length $2 \operatorname{diam}(f(Q))$ which hits $f(Q)$, then $\gamma$ contains a subarc of length $\geq \ell(\gamma) / C$ which is contained in $f(W)$ ( $\ell$ denotes arclength on a rectifiable curve).

Since $f$ is close to linear and an $\epsilon$-Lipschitz graph is close to a line segment, this version can be deduced from Lemma 3.1 as follows. Let $I$ be the line segment with the same endpoints as the curve $\tilde{\gamma}=f^{-1}(\gamma)$. If $\epsilon$ is small enough, the segment and curve are as close as we wish to each other. By our previous argument $I$ hits a square of the form $S_{2}$ and so $\tilde{\gamma}$ comes as close as we wish to such a square, say $1 / 40$, or half the side length of an $S_{1}$ square. Then $\tilde{\gamma}$ has a subarc of diameter $\geq 1 / 40$ inside $W$. By quasisymmetry the image arc $\gamma$ has a subarc of diameter $\sim \ell(\gamma)$ inside $f(W)$, as desired.

We now make use of our parameter $N$, by using a forest with parameter $\epsilon=$ $1 / N$. Suppose $\alpha>4$ (to be fixed later). Now take a $\left(\frac{1}{N}, C, 4\right)$ forest for the plane and rescale it by a factor $\sim N^{-\alpha}$ so that points are separated by at least $r=\ell(Q) /\left(100 C N^{\alpha}\right)$ and intersect it with the interior region $S_{1}$ to get a set $E_{Q}$. The constant $C$ is chosen so that any line segment that hits $S_{2}$ in length $\geq \ell(Q) / 100 N^{\alpha-4}$ must pass within $C \ell(Q) / N^{\alpha+1}$ of one of the points of $E_{Q}$ and that that points in $E_{Q}$ are $\geq N^{-\alpha} / C$ apart.

Form the type II piece associated to $Q$ by dividing $S_{1}$ into equal squares of side length $\ell(Q) / N^{\alpha+4}$. Then remove each square that contains a point of $E_{Q}$ in its closure. What remains is a type II piece.

The point is that these holes are so small that Lemma 2.1 will apply and we can construct rectifiable curves that avoid the holes. Each hole is inside a disk of radius $\sim N^{-\alpha}$ and these disks are disjoint and the distance of each disk from the edge of the tile is $\sim 1$. Thus Lemma 2.1 will apply with $P \sim N^{\alpha}$ and $Q \sim N^{4}$. On the other hand, in each disk we can use a rescaled power function to quasiconformally increase the size of the holes from $N^{-\alpha-4}$ to $N^{-\alpha-1}$ (roughly this leaves $P$ alone and decreases $Q$ from $N^{4}$ to $N$ ). We can then apply Lemma 2.3 to show there is no rectifiable curve in the image set. 
3.3. Definition of $E$ and proof that $E$ has zero area. The set $E$ will depend on a number of parameters: increasing sequences of positive integers $\left\{L_{n}\right\},\left\{M_{n}\right\}$, $\left\{N_{n}\right\}$ and a set of positive integers $S=\left\{n_{k}\right\} \subset \mathbb{N}$. We will see later that it suffices to take

$$
L_{n}=\left\lfloor n^{3 / 4}\right\rfloor, \quad M_{n}=\lfloor\sqrt{n}\rfloor, \quad N_{n}=\left\lfloor n^{\beta}\right\rfloor,
$$

where $\beta$ will be chosen below. We will show later that our example has the desired properties if $\left\{n_{k}\right\}$ grows fast enough. For the present, assume $S$ is enumerated in increasing order and that $0,1, \notin S$ and $2 \mathbb{Z} \cap S=\emptyset$.

Let $s_{0}=1$ and in general let $s_{n}=s_{n-1} /\left(6 L_{n} M_{n}\right)$ if $n \notin\left\{n_{k}\right\}$ and $s_{n}=$ $s_{n-1} / N_{k}^{\alpha+4}$ if $n=n_{k}$. The number $s_{n}$ is the side length of the squares which we replace at stage $n+1$ of the construction.

If $n \notin S$, then we do a type I replacement using a $\left(L_{n}, M_{n}\right)$-piece. Otherwise $n=n_{k}$ for some $k$ and we do a type II replacement using a $N_{k}$-piece. Let $E_{n}$ denote the compact set obtained at step $n$, and let $E=\cap_{n} E_{n}$. Each $E_{n}$ is a union of squares of size $s_{n}$. Moreover, if $n \notin S$, then area $\left(E_{n+1}\right) \leq(1-$ $\left.O\left(M_{n}^{-2}\right)\right) \operatorname{area}\left(E_{n}\right)$ and hence

$$
\operatorname{area}(E) \leq \prod_{n: n \notin S}\left(1-\frac{1}{M_{n}^{2}}\right) \leq \prod_{n \in 2 \mathbb{Z}}\left(1-\frac{1}{M_{n}^{2}}\right)=0,
$$

we can deduce $\operatorname{area}(E)=0$.

3.4. Proof that $E$ contains rectifiable curves QC-locally. It is enough to consider quasiconformal maps which fix 0 and $\infty$. It is clear that condition 1 of Lemma 2.1 holds for both type 1 and type 2 replacement pieces as well as any normalized quasiconformal image of such pieces. Thus we only have to check condition 2 .

For a type I piece, condition 1 of Lemma 2.1 holds for $P_{n} \sim L_{n} \sim n^{3 / 4}$ and $Q_{n} \sim M_{n} \sim n^{1 / 2}$ and hence $\sum P_{n}^{-2} \sim \sum n^{-3 / 2}$ and $\sum Q_{n}^{-3} \sim \sum n^{-3 / 2}$.

For a type II piece, assume $n=n_{k}$ for some $k$ and the the Lemma 2.1 holds with $P_{n} \sim N_{k}^{\alpha} \sim k^{\alpha \beta}$ and $Q_{k} \sim N_{k}^{-\alpha} / N_{k}^{-\alpha-4} \sim N_{k}^{4} \sim k^{4 \beta}$. Hence $\sum P_{n}^{-2} \sim \sum k^{-2 \alpha \beta}$ and $\sum Q_{n}^{-3} \sim \sum k^{-12 \beta}$. Both these sums converge if $-2 \alpha \beta<-1$ and $-12 \beta<-1$, so we want $\beta>1 / 12$ and $\alpha>1 /(2 \beta)$. If so, then Lemma 2.1 applies to $E$ and $E$ contains rectifiable curves. 
Moreover, a $K$-quasiconformal map is quasisymmetric. In particular, if $K$ is close to 1 then

$$
\left(\frac{|x-y|}{|x-z|}\right)^{1+\eta} \leq \frac{|f(x)-f(y)|}{|f(x)-f(z)|} \leq\left(\frac{|x-y|}{|x-z|}\right)^{1-\eta},
$$

with $\eta$ close to 0 . This means the ratios $P_{n}$ and $Q_{n}$ can only change by a small power. Thus the relevant series still converge for any quasiconformal image with small enough constant. This proves $E$ contains rectifiable curves QC-locally.

3.5. A QC-image of $E$ with no rectifiable subcurve. To produce a quasiconformal image of $E$ which contains no rectifiable curves, we want define a map which increases the size of the omitted squares in the special generations $\left\{n_{k}\right\}$ by a power. For $0<\rho<1$, consider the map

$$
f_{\rho}(z)= \begin{cases}z, & \text { if }|z| \geq 1 \\ z|z|^{\rho-1}, & \text { if } \epsilon<|z|<1 \\ z \epsilon^{\rho-1}, & \text { if }|z| \leq \epsilon .\end{cases}
$$

This map is $K$-quasiconformal with $K=1 / \rho$ and maps the disk $D(0, \epsilon)$ to the disk $D\left(0, \epsilon^{\rho}\right)$. Suppose $n=n_{k} \in S$ is a generation where we use a type II replacement in a square of size $s=s_{n}$. Let $N=N_{k}$. Then the omitted squares are contained in disjoint disks of radius $\simeq s / N^{\alpha}$ and each of them have diameter $\simeq s / N^{\alpha+4}$. Let this collection of disks be denoted $\mathcal{C}$. In each of the disjoint disks we can apply a rescaled version of the map $f_{\rho}$ with $\rho=1 / 4$ to increase the size of the omitted square to $s / N^{\alpha+1}$. Since the squares are centered on a forest, a line segment of length $s / N^{\alpha-4}$ will hit a disk contained in some enlarged omitted square. Take $\beta=1 / 10$ and $\alpha=21>2 / \beta=20$. This is consistent with our previous requirements and if we set $a_{k}=k^{-5 \beta}=1 / \sqrt{k}$, then $\sum a_{k}^{2}=\infty$.

Assume for the moment that we can construct a quasiconformal map $f$ so that on any bent tile of any generation, the map $f$ looks like $f_{\rho}$, i.e., $f=L \circ\left(f_{\rho}+\right.$ $\left.o\left(s / N^{\alpha+1}\right)\right)$, where $L$ is conformal and linear. Next, suppose $f(E)$ contains a rectifiable curve $\Gamma$. We will show this leads to a contradiction.

By Lemma 2.7 there is an $\epsilon$-Lipschitz graph $\gamma$ which intersects $\Gamma$ in positive length. Suppose $Q$ is a bent tile in generation $n_{k}$ and $\gamma$ hits $f(Q)$. Let $W_{k}$ be the union of "tile interiors", as in Lemma 3.1, where the union is over all tiles corresponding to an integer grid rescaled to side length $s=s_{n_{k}}$. Then by Lemma 
3.2 any subarc $I$ of $\gamma$ which hits the image $f(Q)$ of a tile $Q$ and has length $\geq 2 \operatorname{diam} f(Q)$ must contain a subarc $J \subset I \subset \gamma$ with $\ell(J) \sim \ell(I)$ and $J \subset f\left(W_{k}\right)$.

Let $E_{k} \subset \gamma$ be the union of these subarcs (where the union is taken over all tiles hit by $\gamma$ ). Note that $E_{k}$ takes up a fixed fraction of any arc on $\gamma$ which hits some $f(Q)$ and has length $\gg \operatorname{diam}(f(Q))$. Since the scales $\left\{s_{n_{k}}\right\}$ decrease rapidly, this implies that $E_{k}$ takes up a fixed fraction of the length of any component of $E_{j}$ or $\gamma \backslash E_{j}$ for $j<k$. Thus we can apply Lemma 2.5 and deduce that there is a set $X$ of full measure in $\gamma$ such that each point of $X$ is in $f\left(W_{k}\right)$ for a positive density set of $k$ 's.

If $Q$ is a $n_{k}$ th generation tile and $x \in \gamma \cap f\left(Q \cap W_{k}\right)$, then because $f$ approximates $f_{\rho}$ composed with a linear map with error less than the size of the holes, we deduce that any segment of length $\operatorname{diam}(f(Q)) / N^{\alpha-4}$ hits a ball of radius $\operatorname{diam}(f(Q)) / N^{\alpha+1}$ which misses $f(E)$. Thus if we take $a_{k}=N_{k}^{-\alpha-1}=$ $N_{k}^{-5}=1 / \sqrt{k}$, then $\sum a_{k}^{2}=\infty$ and Lemma 2.5 implies that $\gamma \cap f(E)$ must have zero length. This is a contradiction, so we deduce that $f(E)$ cannot contain any rectifiable curve.

Finally, we have to show that $f$ approximates $f_{\rho}$ as claimed. If we only had to worry about one generation of type II tiles there would be nothing to do, since then we would have $f=f_{\rho}$. However, when we define these power maps for more than one generation some errors are introduced. Thus we need only see that the errors introduced by other generations are as small as we wish if the gaps in the set $S=\left\{n_{k}\right\}$ are sufficiently large.

The first error arises as follows. To keep the quasiconformal constant bounded, we want to arrange for supports of the complex dilatations of maps from different generations to be disjoint. We do this by replacing $f_{\rho}$ by a quasiconformal approximation, $g_{\rho}$, whose dilatation is supported away from $E$. More precisely, let $F_{n}$ be a $s_{n}$-neighborhood of $E_{n}$. Then $\cap_{n} F_{n}=E$ and area $\left(F_{n}\right) \leq 2 \operatorname{area}\left(E_{n}\right) \rightarrow 0$. Define an approximation $g_{\rho}$ to $f_{\rho}$ in each square by restricting its dilatation to the complement of $F_{n_{k+1}}$. Since area $\left(F_{n}\right) \rightarrow 0$, we have $g_{\rho} \rightarrow f_{\rho}$ uniformly (with an estimate depending on $\rho$, but not on $f$ ). Thus we can choose $n_{k+1}$ so large that $\left\|f-g_{\alpha}\right\|_{\infty} \ll s_{n_{k}}$. Thus this type of error is small if the gaps are large.

The second type of error comes from the maps defined at earlier stages. We just saw that we choose maps $g_{m}$ which are conformal on a neighborhood of $E$ and hence conformal on a neighborhood of the $n$th generation squares if $n$ is large 


\section{A Set Containing Rectifiable Arcs QC-locally But Not QC-globally 137}

enough. In fact, this map will be close to linear on such squares because their diameter is very small compared to the distance to the support of $g_{\rho}$. In fact, we may make this ratio as large as we wish by choosing $n_{k+1}$ large enough with respect to $n_{k}$.

The final type of error comes from later generations of maps. However, the map $g_{\rho}$ for generation $n_{k}$ is supported inside $E_{n_{k}}$, which has area tending to zero as $n_{k+1} \nearrow \infty$, so that the corresponding conformal maps approximate the identity at any fixed scale as $n_{k+1} \rightarrow \infty$. In particular we can choose $n_{k+1}$ so large that the perturbations at scale $s_{n_{k}}$ are small compared to $s_{n_{k}} / N_{k}^{\alpha+1}$, which is what we needed. This completes the proof of Theorem 1.1.

\section{REFERENCES}

[1] C. J. Bishop. An $A_{1}$ weight not comparable to any quasiconformal Jacobian. In the tradition of Ahlfors-Bers. IV, Contemp. Math., Amer. Math. Soc. 432:7-18, 2007.

[2] C. J. Bishop and P. W. Jones. Harmonic measure and arclength. Ann. of Math. (2), 132(3):511-547, 1990.

[3] C. J. Bishop and P. W. Jones. Harmonic measure, $L^{2}$ estimates and the Schwarzian derivative. J. Anal. Math., 62:77-113, 1994.

[4] M. Bonk, J. Heinonen, and E. Saksman. The quasiconformal Jacobian problem. Contemp. Math., 355:77-96, 2004.

[5] J. W. S. Cassels. An introduction to Diophantine approximation. Cambridge Tracts in Mathematics and Mathematical Physics, No. 45. Cambridge University Press, New York, 1957.

[6] J. B. Garnett and D.E. Marshall. Harmonic measure. New Mathematical Monographs, 2. Cambridge University Press, 2005.

[7] J. Heinonen. The branch set of a quasiregular mapping. In Proceedings of the International Congress of Mathematicians, Vol. II (Beijing, 2002), pages 691-700, Beijing, 2002. Higher Ed. Press.

[8] J. Heinonen. Geometric embeddings of metric spaces. 2003. Jyväskylä Mathematics Department Reports.

[9] J. Heinonen and S. Semmes. Thirty-three yes or no questions about mappings, measures, and metrics. Conform. Geom. Dyn., 1:1-12 (electronic), 1997.

[10] P. W. Jones. Rectifiable sets and the traveling salesman problem. Invent. Math., 102(1):1$15,1990$.

[11] P. W. Jones. The traveling salesman problem and harmonic analysis. Publ. Mat., 35(1):259267, 1991. Conference on Mathematical Analysis (El Escorial, 1989).

[12] K. Okikiolu. Characterization of subsets of rectifiable curves in $\mathbf{R}^{n}$. J. London Math. Soc. (2), 46(2):336-348, 1992.

[13] S. Semmes. Bi-Lipschitz mappings and strong $A_{\infty}$ weights. Ann. Acad. Sci. Fenn. Ser. A I Math., 18(2):211-248, 1993. 
Christopher Bishop

Mathematics Department,

SUNY at Stony Brook,

Stony Brook, NY 11794-3651

E-mail: bishop@math.sunysb.edu 